\title{
How to prevent the infection of contaminated abdominal incisions
}

\author{
Hao Wang ${ }^{\mathrm{a}}$, Xin-Yuan Li ${ }^{\mathrm{b}}$, Yan-Ming $\mathrm{An}^{\mathrm{a}}$ and Ya-Min Guo ${ }^{\mathrm{a}, *}$ \\ ${ }^{a}$ Department of General Surgery, People's Hospital of Qinghai Province, Xining, Qinghai, China \\ ${ }^{\mathrm{b}}$ Qinghai University Graduate College, Xining, Qinghai, China
}

\begin{abstract}
.
BACKGROUND: Surgical site infection is one of the most common complications of conventional laparoscopic surgery. Preventing infection of the incision is particularly important.

OBJECTIVE: To discuss how to prevent the occurrence of surgical site infection after contaminated abdominal surgery.

METHODS: Five hundred and fifty-one surgery patients with "contaminated abdominal incisions" from January 2011 to May 2013 were analyzed in terms of the preventative treatment, and summarized for surgical site infection. Subcutaneous tissue flushed with normal saline + hydrogen peroxide before suturing in the intervention 1 group; subcutaneous tissue flushed with normal saline $+0.5 \%$ povidone-iodine before suturing in the intervention 2 group.

RESULTS: When subcutaneous fat was contaminated to a depth of $\leqslant 2.5 \mathrm{~cm}$, the rates of surgical site infection in the control group and the intervention groups showed no significant difference $(P>0.05)$. When subcutaneous fat was contaminated to a depth of $\geqslant 3.0 \mathrm{~cm}$, the rate of surgical site infection in the control group compared with the intervention one group was not statistically different $(P>0.05)$. The rate of surgical site infection in the control group compared with the intervention two group was statistical significant $(P<0.05)$. The rate of surgical site infection in the intervention one group compared with the intervention two group was statistical significant $(P<0.05)$.

CONCLUSIONS: Preoperative control of the blood sugar; correction of anemia and the hypoalbuminemia; use of intraoperative the high-frequency electrotome; irrigation of the incision with plenty of physiological saline + iodophor before suturing the subcutaneous fat layer were key to effectively preventing infection in contaminated abdominal incisions.
\end{abstract}

Keywords: Surgical site infection, preventive treatment

\section{Introduction}

Surgical site infection (SSI) is one of the most common complications of conventional laparoscopic surgery. Patients experience more pain which adds to the economic burden [1] to both the patient and the hospital. It is for these reasons that the prevention and treatment of SSI has become crucial. If effective, simple and feasible measures are undertaken in the surgical process, the risk of SSI would be significantly lower.

\section{Materials and methods}

\subsection{Research object}

Between January 2011 and May 2013551 patients from our hospital were selected as research objects. The intraoperative diagnoses were as follows: acute purulent appendicitis (156 cases), acute gangrenous

\footnotetext{
${ }^{*}$ Corresponding author: Ya-Min Guo, Department of General Surgery, People's Hospital of Qinghai Province, No. 2, Gonghe road, Chengdong District, Xining, Qinghai, China. Tel.: +86 13519763975; E-mail: wanghao840323@163.com.
}

0928-7329/16/\$35.00 (c) 2016 - IOS Press and the authors. All rights reserved This article is published online with Open Access and distributed under the terms of the Creative Commons Attribution NonCommercial License. 
appendicitis (103 cases), periappendiceal abscess (52 cases), obstruction with strangulation (74 cases), acute obstructive suppurative cholangitis (78 cases), gastrointestinal perforation (27 cases), digestive tract injury after abdominal stab wound ( 34 cases), severe acute pancreatitis with acute diffuse peritonitis ( 8 cases), restorative surgery after enterostomy ( 9 cases), postoperative biliary fistula ( 3 cases) and other ( 7 cases). The mean of age of patients was $37.6 \mathrm{y}$. The number of patients whose subcutaneous fat thickness $\leqslant 2.5 \mathrm{~cm}$ was 263 , while number of the patients with subcutaneous fat thickness $\geqslant 3.0 \mathrm{~cm}$ was 288 . The preoperative examination confirmed that the selected patients did not have nasopharyngeal infection, chronic respiratory tract infection, skin infections, diabetes, liver cirrhosis, lung disease, severe hypoalbuminemia or hemophilia.

\subsection{The diagnosis of incision infection [2]}

The following criteria were used to consider whether the patients had an incision infection: patients have a fever of unknown origin with a sustained temperature $\left(\geqslant 38^{\circ}\right) 4-6$ days afer operation or the temperature temporarily normalizes then increased, feelings of unexplained intensified pain in the incision area, abnormal seepage during dressing change, feelings of tenderness at the site of incision, pitting edema, secretions in poorly healing areas, foul smelling incisions, phlegmon by microscopic examination, bacterial culture showing escherichia coli, anaerobic bacteria (bacteriodes fragilis), staphylococcus aureus, hemolytic streptococcus.

\subsection{Grouping and intervention methods in the operation}

The cases were divided into 3 groups: subcutaneous tissue flushed with normal saline before suturing in the control group; subcutaneous tissue flushed with normal saline + hydrogen peroxide before suturing in the intervention group 1 ; subcutaneous tissue flushed with normal saline $+0.5 \%$ povidone-iodine before suturing in the intervention group 2 . The patients with subcutaneous fat thickness $\leqslant 2.5 \mathrm{~cm}$ were randomly divided into these three groups, 74 cases in the control group, 82 cases in the intervention group 1, 107 cases in the intervention group 2; The patients with subcutaneous fat thickness $\geqslant 3.0 \mathrm{~cm}$ were randomly divided into the three groups, 87 cases in the control group, 76 cases in the intervention group 1,125 cases in the intervention group 2 . The patients with subcutaneous fat thickness $\leqslant 2.5 \mathrm{~cm}$ were single-layer sutured; the patients with subcutaneous fat thickness $\geqslant 3.0 \mathrm{~cm}$ were double-layer sutured then the skin was suture closed.

\subsection{Outcome measures}

The same parameters that were used for patient inclusion were used as outcome measures (Section 2.2).

\subsection{Statistical methods}

We used statistical software SPSS 18.0 to calculate and compared data using $X^{2}$ test, compared between groups using $X^{2}$ segmentation test, inspection level alpha $=0.05$. 
Table 1

Rates of SSI in subcutaneous fat thickness $\leqslant 2.5 \mathrm{~cm}$ groups

\begin{tabular}{lcccccc}
\hline Groups & Incision infection & No incision infection & Total & Incision infection rate & $X^{2}$ & $P$ \\
\hline Control group & 4 & 70 & 74 & $5.4 \%$ & 0.850 & $>0.05$ \\
Intervention group 1 & 7 & 75 & 82 & $8.5 \%$ & & \\
Intervention group 2 & 6 & 101 & 107 & $5.6 \%$ & & \\
Total & 17 & 246 & 263 & & & \\
\hline
\end{tabular}

Table 2

Rates of SSI in subcutaneous fat thickness $\geqslant 3.0 \mathrm{~cm}$ groups

\begin{tabular}{lcccccc}
\hline Groups & Incision infection & No incision infection & Total & Incision infection rate & $X^{2}$ & $P$ \\
\hline Control group & 20 & 67 & 87 & $29.8 \%$ & 19.872 & $<0.05$ \\
Intervention group 1 & 17 & 59 & 76 & $22.3 \%$ & & \\
Intervention group 2 & 5 & 120 & 125 & $4.0 \% \%^{* * * *}$ & & \\
Total & 42 & 246 & 288 & & & \\
\hline
\end{tabular}

Compared with control group ${ }^{*} P<0.05$, compared with the intervention group $1^{* *} P<0.05$.

Table 3

Rates of SSI in subcutaneous fat $\leqslant 2.5 \mathrm{~cm}$ compared with subcutaneous fat $\geqslant 3.0 \mathrm{~cm}$ in different groups

\begin{tabular}{|c|c|c|c|c|c|c|c|c|c|c|c|c|}
\hline $\begin{array}{l}\text { Subcutaneous } \\
\text { fat }\end{array}$ & Group & Case & $\begin{array}{l}\text { Incision } \\
\text { infection } \\
\text { rate }\end{array}$ & $P$ & Group & Case & $\begin{array}{l}\text { Incision } \\
\text { infection } \\
\text { rate }\end{array}$ & $P$ & Group & Case & $\begin{array}{l}\text { Incision } \\
\text { infection } \\
\text { rate }\end{array}$ & $P$ \\
\hline$\leqslant 2.5 \mathrm{~cm}$ & $\begin{array}{l}\text { Control } \\
\text { group }\end{array}$ & 74 & $5.4 \%$ & $<0.05$ & $\begin{array}{l}\text { Intervention } \\
\text { group } 1\end{array}$ & 82 & $8.5 \%$ & $<0.05$ & $\begin{array}{l}\text { Intervention } \\
\text { group } 2\end{array}$ & 107 & $5.6 \%$ & $>0.05$ \\
\hline$\geqslant 3.0 \mathrm{~cm}$ & & 87 & $29.8 \%$ & & & 76 & $22.3 \%$ & & & 125 & $4.0 \%$ & \\
\hline
\end{tabular}

\section{Results}

\subsection{The result of subcutaneous fat thickness $\leqslant 2.5 \mathrm{~cm}$}

The rates of SSI in control group, intervention group 1 and intervention group 2 were compared with each other. There was no statistical difference between the 3 groups $(P>0.05)$. See Table 1 .

\subsection{The results of subcutaneous fat thickness $\geqslant 3.0 \mathrm{~cm}$}

The rate of SSI in the control group compared with intervention group 1 was not statistically significant $(P>0.05)$. The rate of SSI in the control group compared with intervention group 2 was statistical significant $(P<0.05)$. The rate of SSI in intervention group 1 compared with intervention group 2 was statistically significant $(P<0.05)$. See Table 2 .

\subsection{The results of rates of SSI in subcutaneous fat $\leqslant 2.5 \mathrm{~cm}$ compared with subcutaneous fat $\geqslant 3.0 \mathrm{~cm}$ in different groups}

The rate of SSI in the control group (subcutaneous fat $\leqslant 2.5 \mathrm{~cm}$ ) compared with control group (subcutaneous fat $\geqslant 3.0 \mathrm{~cm})$ was statistically significant $(P<0.05)$. The rate of SSI in the intervention group 1 (subcutaneous fat $\leqslant 2.5 \mathrm{~cm}$ ) compared with intervention group 1 (subcutaneous fat $\geqslant 3.0 \mathrm{~cm}$ ) was statistically significant $(P<0.05)$. The rates of SSI in intervention group 2 (subcutaneous fat $\leqslant$ $2.5 \mathrm{~cm}$ ) compared with intervention group 2 (subcutaneous fat $\geqslant 3.0 \mathrm{~cm}$ ) was statistically significant $(P>0.05)$. See Table 3 . 


\section{Discussion}

SSI is the most common complication after surgeries where the incision is contaminated. Important causes of SSI include bacterial infection, poor host defenses, subcutaneous fat aseptic necrosis, secondary infection, etc. Operators can effectively prevent and treat SSIs by determining the source of infection, eliminating the pathway of incision infection, improving local environmental factors, improving host defense capabilities and reducing subcutaneous fat aseptic necrosis.

\subsection{Causes for SSI}

The rate of SSI in contaminated incisions is seven times higher than that of clean incisions [3]. Ineffective skin shaving, absense of standardized hand-washing, incomplete disinfection, smaller scopes of disinfection fields, violating operation regulations, long operation time, too many post of visitors, incorrect and frequent using of an electric knife, dead space or foreign bodies in the subcutaneous tissue and uncontrolled intra-abdominal infection all contribute, Host factors such as diabetes, malnutrition, poor immune function, alcohol poisoning, malignant tumor, organ transplantation and lung diseases are potential contributors to incision infection $[4,5]$. This study illustrates the SSIs are closely correlated to the thickness of subcutaneous fat layer, which were consistent with the report of Watanabe [6,7]. The risk of SSI can be induced by flushing the contaminated abdominal incisions. Aseptic necrosis of adipose tissue is a local factor in poor healing, and also contributes to secondary infection.

\subsection{Prevention and treatment of SSI}

\subsubsection{Prevention of SSI}

The main way to prevent an SSI is during preoperative and intraoperative care. The risk of SSIs is lessened by shortening the preoperative hospitalization time, reducing the chance of nosocomial cross infection, treating anemia and hypoalbuminemia, improving immune system of patients, controlling the blood glucose, using aseptic technique, shortening the operation time, reducing the length of incision, reducing the exposure time of incision and the contact area with bacteria, using control of the high frequency electricity knife, avoiding repeated cutting and contacting of adipose tissue, avoiding high temperatures which lead to capillary occlusions and avoiding adipose aseptic necrosis. If the infection in the abdominal cavity is serious and purulent exudate is widespread, the abdominal cavity should be flushed thoroughly before closing, however if is not recommended to flush the abdominal cavity for suppurative appendicitis or acute appendicitis with perforation because this can spread the infection. It is important to drain the abdomen sufficiently, so as to avoid reinfection. It is recommended to change the gloves, the aspirator and other used instruments (or disinfect used instruments with iodine volts, alcohol) and flush the incision with a large volume of physiological saline $+0.5 \%$ iodine volts or hydrogen peroxide before suturing the subcutaneous fat layer [6]. It is not recommended to suture the thin fat layer (subcutaneous fat $\leqslant 1.0 \mathrm{~cm}$ ) of patients because it can reduce the blood supply. It is recommended to put homemade double-cavity catheter in the incision and keep the drainage catheter to negative pressure for the thick fat layer (subcutaneous fat $\geqslant 3.0 \mathrm{~cm}$ ) of patients. This method can reduce effusion, hemorrhage, the rate of SSI and also can be used to flush the infected incision after operation. Alcohol dissolves and liquefies fat, therefore it is not recommended to put alcohol soaked gauze on the incision after suturing. It is instead recommended to put that the non liposoluble liquid, such as iodine, soaked gauze on the incision. 


\subsubsection{The treatment of SSI}

When an SSI is present and the inflammatory exudate is minimal, it is recommended to remove 1 or 2 stitches and remove the ligation suture in fat layer. It is recommended to remove the stitches in the rectus abdominis anterior sheath in 9-10 days after operation and fill the deep incision with $10 \%$ hypertonic saline gauze for sufficiently draining. It is not wise to place large amount of gauze in the incision because this lessens the drainage and prevents the growth of granulation tissue. It is recommended to open the incision in a limited fashion, maintain unobstructed drainage, use hypertonic saline gauze to drain the inflammatory exudate and place a secondary suture after the formation of fresh granulation tissue. It is effective to place a multihole drainage catheter into the incision, then keep the drainage catheter to negative pressure [8]. It can take the place of secondary suture that we put a double-cavity catheter into the incision when the incision is infection and deep, flush the incision with physiological saline for 23 days, intermittently flush when the drainage is clear, in the end gradually remove the double-cavity catheter. Electromagnetic heat will keep the incision dry and reduce the exudate. It is doubtful how effective the use of antibiotics is in the treatment of SSI. To avoid bacteria resistance, we recommend not to put antibiotics directly into an incision. Using broad-spectrum antimicrobials within 30 minutes before operation, and applying sensitive antimicrobials according to the results of bacterial culture is best for the prevention of incision infection $[9,10]$.

In conclusion, there are many factors that influence incision healing. We must integrate a variety of measures in order to prevent SSIs. These results show that SSIs can effectively be prevented in contaminated abdominal incisions by flushing the incisions with physiological saline $+0.5 \%$ iodine or hydrogen peroxide before suturing the subcutaneous fat layer while attending to the patient's nutritional and immune factors. It is effective especially in obese patients.

\section{References}

[1] Maier S, Körner P, Diedrich S, et al. Definition and management of wound infections. Chirurg. 2011 Mar; 82(3): 235-41.

[2] Xh Xu. Lin Chuang Gan Ran Yi Xue. Chang sha: Hunan Science and Technology Press, 2005: 74-76.

[3] Motie MR, Ansari M, Nasrollahi HR. Assessment of surgical site infection risk factors at Imam Reza hospital, Mashhad, Iran between 2006 and 2011. Med J Islam Repub Iran. 2014 Jul 8; 28: 52.

[4] Smith MA, Dahlen NR, Bruemmer A, et al. Clinical practice guideline surgical site infection prevention. Orthop Nurs, 2013; 32(5): 242-248.

[5] Sehgal R, Berg A, Figueroa R, et al. Risk factors for surgical site infections after colorectal resection in diabetic patients. J Am Coll Surg. 2011 Jan; 212(1): 29-34.

[6] Watanabe M, Suzuki H, Nomura S, et al. Risk factors for surgical site infection in emergency colorectal surgery: A retrospective analysis. Surg Infect (Larchmt). 2014 Jun; 15(3): 256-261.

[7] Han J, Wang Z, Wei G, et al. Risk factors associated with incisional surgical site infection in colorectal cancer surgery with primary anastomosis. Zhonghua Wai Ke Za Zhi. 2014 Jun; 52(6): 415-419.

[8] Webb LX, Schmidt U. Wound management with vacuum therapy. Unfallchirurg. 2001 Oct; 104(10): 918-26.

[9] Sumiyama Y, Arima Y. Surgical site infection (SSI) and postoperative infection. Masui. 2010 Jan; 59(1): 36-45.

[10] Anderson DJ. Surgical site infections. Infect Dis Clin North Am. 2011 Mar; 25(1): 135-53. 